Vol. I, $\mathbf{n}^{\mathbf{0}} \mathbf{1}$

Enero 2020

\title{
Know and Share Psychology
}

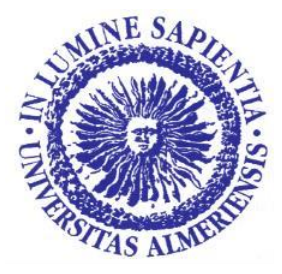

\section{PROPUESTA EDUCATIVA PARA LOS ALUMNOS DE PMAR A TRAVÉS DE LA METODOLOGÍA APRENDIZAJESERVICIO}

\section{EDUCATIONAL PROPOSAL FOR PMAR STUDENTS THROUGH THE LEARNING-SERVICE METHODOLOGY}

\author{
AUTORA \\ NEREA GARCÍA PÉREZ \\ TRABAJO FIN DE GRADO \\ Grado en Psicología \\ Universidad de Almería \\ Almería. España
}

Correspondencia: NEREA GARCÍA PÉREZ. E-mail: nereaperezgarcia1997@gmail.com

(C) Universidad de Almería. Almería. España. 


\title{
RESUMEN
}

Basándonos en la metodología de Aprendizaje y Servicio, presentamos un proyecto de innovación enfocado a optimizar el desarrollo personal, conductual y el rendimiento académico del alumnado de $2^{\circ}$ que cursa el Programa de Mejora Aprendizaje y Rendimiento, en adelante PMAR; no obstante involucraremos al resto del alumnado del centro, a profesores, familias, así como a un agente externo a la comunidad educativa: el Secretariado Gitano de Almería.

El proyecto se realizará en varias sesiones durante el horario de tutoría, cuyas actividades estarán relacionadas con los usos adecuados de la aplicación de Instagram.

Nuestro objetivo es empoderar al alumnado de PMAR a través de dicha metodología, comprobando para ello si la capacidad de resiliencia y autoconcepto del alumnado ha mejorado tras realizar nuestra propuesta educativa a través de la Escala de Resiliencia de Connor y Davidson, CD-RISC, (Connor y Davidson, 2003) y del cuestionario de Autoconcepto Dimensional (AUDIM) de Fernández- Zabala, Goñi, Rodríguez-Fernández y Goñi (2015), respectivamente.

\begin{abstract}
Based on the Learning and Service methodology, we present an innovation project focused on optimizing the personal, behavioral and academic performance of 2nd graders who take the Learning and Performance Improvement Program, going forward PMAR; however, we will involve the rest of the students of the center, teachers, families, as well as an agent external to the educational community: the Gypsy Secretariat of Almería.

The project will be carried out in several sessions during tutoring hours, whose activities will be related to the appropriate uses of the Instagram app.

Our goal is to empower PMAR students through this methodology, checking whether the resilience and self-concept capacity of the students has improved after making our educational proposal through the Connor Resilience Scale and Davidson, CD-RISC, (Connor and Davidson, 2003) and the Dimensional Self Concept (AUDIM) questionnaire by Fernández- Zabala, Goñi, Rodríguez-Fernández and Goñi (2015), respectively.
\end{abstract}

\section{INDICE}

\section{JUSTIFICACIÓN}

PRIMERA PARTE: Marco teórico.

SEGUNDA PARTE: Propuesta educativa

- Contextualización

- Objetivos

- Contenidos

- Metodología

- Cronograma

- Recursos

- Resultados esperados

REFERENCIAS BIBLIOGRÁFICAS 


\section{JUSTIFICACIÓN}

Existe un gran interés por lograr una mejora en la calidad del sistema educativo español, tal y como afirma Barrio (2006). De hecho una de las iniciativas del Consejo de la Unión Europea (Ministerio de Educación y Formación Profesional, 2018) sobre el estado del sistema educativo en España, fue estudiar una serie de medidas para promover el protagonismo y la participación de los alumnos/as en los distintos centros escolares con el fin de mejorar su experiencia educativa, dando una gran importancia al papel de los/as estudiantes como agentes activos para mejorar los sistemas educativos y así alcanzar una educación de alta calidad para TODOS.

Hoy día la diversidad del alumnado en nuestras aulas es una realidad, tal y como viene indicado en el artículo 14. Alumnado con necesidades específicas de apoyo educativo, podemos encontrar un alumnado que requiera una atención educativa diferente a la ordinaria, por presentar necesidades educativas especiales, tales como dificultades específicas de aprendizaje, Trastorno por Déficit de Atención e Hiperactividad (TDAH), por sus altas capacidades intelectuales, por haberse incorporado tarde al sistema educativo, o por condiciones personales o de historia escolar.

Efectivamente el Sistema Educativo debe de dar una respuesta de calidad a todos los alumnos. Un alumnado muy especial es el que participa en los Programas de Mejora del Aprendizaje y Rendimiento (PMAR, en adelante).

Una propuesta para mejorar la calidad de los/as alumnos/as sería utilizar la metodología de Aprendizaje y Servicio (APS, en adelante). Cada vez son más numerosos los resultados de investigación que muestran los beneficios del APS (Puig, Batle, Bosch y Palos (2007); Furco y Billig (2002); Martínez, Martínez, Alonso y Geruzaga (2013) entro otros) para el alumnado que participa en ellos.

Recordemos que estos/as alumnos/as que pertenecen a P.M.A.R, utilizan una metodología específica a través de la reorganización de las materias y contenidos, con el fin de que lleguen a $4^{\circ}$ ESO y obtengan el título de G.E.S.O.

Los cursos de PMAR, tal y como afirman Corujo, Méndez y Rodríguez (2018) presentan una serie de características como son:

- Se desarrolla a partir de $2^{\circ}$ curso de la ESO y tiene una duración de dos años. Excepcionalmente, aquellos alumnos y alumnas que, habiendo cursado $3^{\circ} \mathrm{de}$ Educación Secundaria Obligatoria, no estén en condiciones de promocionar a $4^{\circ}$, podrán incorporarse a un Programa de mejora del aprendizaje y del rendimiento para repetir 3er curso.

- Utilizarán una metodología específica, a través de la organización de contenidos, actividades prácticas y, en su caso, de materias diferentes a las establecidas con carácter general.

- Tiene la finalidad de que los alumnos y alumnas puedan cursar $4^{\circ}$ curso por la vía ordinaria y obtengan el título de Graduado en Educación Secundaria Obligatoria. 
- Se dirige al alumnado que presente dificultades relevantes de aprendizaje no imputables a falta de estudio o esfuerzo. También para el alumnado con "discapacidad".

- El equipo docente podrá proponer a los padres, madres o tutores legales la incorporación a un Programa de mejora del aprendizaje y del rendimiento de aquellos alumnos y alumnas que hayan repetido, al menos, un curso en cualquier etapa y que, una vez cursado $1^{\circ}$ de ESO, no estén en condiciones de promocionar a $2^{\circ}$ curso, o que una vez cursado $2^{\circ}$, no estén en condiciones de promocionar a $3^{\circ}$.

- En todo caso, su incorporación requerirá la evaluación, tanto académica como psicopedagógica, y la intervención de la Administración educativa en los términos que ésta establezca; y se realizará una vez oídos los propios alumnos o alumnas y sus padres, madres o tutores legales.

- Se potenciará la acción tutorial como recurso educativo que pueda contribuir, de una manera especial, a subsanar las dificultades de aprendizaje y a atender las necesidades educativas del alumnado.

- La evaluación tendrá como referente fundamental las competencias y los objetivos de la Educación Secundaria Obligatoria, así como los criterios de evaluación y los estándares de aprendizaje evaluables.

De forma general podemos decir que son alumnos/as que presentan un déficit importante de conocimientos, dificultades para autorregular su aprendizaje, desmotivación en el aula producto de la inexistencia de buenas notas en las asignaturas, un autoconcepto negativo de ellos mismos tanto a nivel personal como académico debido a las numerosas comparaciones a las que se enfrentan día a día con el resto de sus compañeros/as de la ESO y todo ello se traduce en una alta tasa de absentismo y una gran desorientación de su vida académica.

En este Trabajo Fin de Grado (TFG, en adelante) elaboraremos una propuesta educativa, basada en la metodología de Aprendizaje y Servicio (Mayor y Rodríguez, 2016), cuyo fin es empoderar al alumnado de PMAR otorgándole un papel protagonista. El objetivo principal de este proyecto es optimizar el desarrollo personal, conductual y rendimiento académico de los/as alumnos/as de $2^{\circ}$ de PMAR. Por otro lado, evaluaremos si la capacidad de resiliencia y autoconcepto del alumnado ha mejorado tras realizar nuestra propuesta educativa a través de la Escala de Resiliencia de Connor y Davidson, CD-RISC, (Connor y Davidson, 2003) y del cuestionario de Autoconcepto Dimensional (AUDIM) de Fernández- Zabala, Goñi, Rodríguez-Fernández y Goñi (2015), respectivamente.

\section{PRIMERA PARTE: MARCO TEÓRICO: APROXIMACIÓN CONCEPTUAL A LA METODOLOGÍA DE APRENDIZAJE Y SERVICIO 1.CONCEPTO DE APRENDIZAJE Y SERVICIO}

Según Folgueiras, Luna y Puig (2014) a nivel europeo son escasas las investigaciones en secundaria sobre el Aprendizaje y Servicio (APS). Sin embargo, el desarrollo de nuevos proyectos en centros educativos es una realidad que está creciendo en los últimos años. En España podemos encontrar los Premios Aprendizaje-Servicio, que son una iniciativa conjunta de tres organizaciones: la Red Española de Aprendizaje-Servicio, la ONG Educo y la Editorial Edebé. El objetivo que se persigue con ello es triple: 1) Reconocer la labor de los educadores, centros educativos y entidades sociales que promueven el aprendizaje-servicio. 2) 
Dar voz y protagonismo a los niños, niñas y jóvenes comprometidos, en particular a aquellos con menores oportunidades. 3) Expandir esta metodología educativa. (Recogido de la página web: https://aprendizajeservicio.net/premios-aps-2018/)

Mayor y Rodríguez (2016) afirman que, en Cataluña, el APS, actualmente se ha implantado en los centros educativos como un servicio obligatorio a la comunidad dentro de los planes de estudio de la Educación Secundaria Obligatoria.

Pero ¿cómo surge este interés por indagar en las actividades de aprendizaje y servicio? Surgen según Puig, Gijón, Martín y Rubio (2011) a raíz de la mirada inquieta y atenta a la comunidad. Si bien la clave de su originalidad tal y como afirman Folgueiras et al. (2014) radica en una metodología que implica la interrelación de dos elementos clave: el Aprendizaje Curricular y el Servicio a la Comunidad. Según Puig et al. (2007) dicha unión supondrá que el servicio dado se vinculará con los cursos reglados, proporcionando un mayor significado y ofreciéndoles a los/as alumnos/as experiencias sobre las que reflexionar. Y a la inversa, el contenido de los cursos se verá enriquecido al ser utilizado durante la realización del servicio. Produciendo de este modo unos resultados en el alumnado que no son sencillos de obtener si utilizamos otros caminos.

No debemos confundir el APS con otras prácticas experienciales, tal y como especifica Puig et al. (2007) como el trabajo de campo, las iniciativas solidarias o los servicios comunitarios.

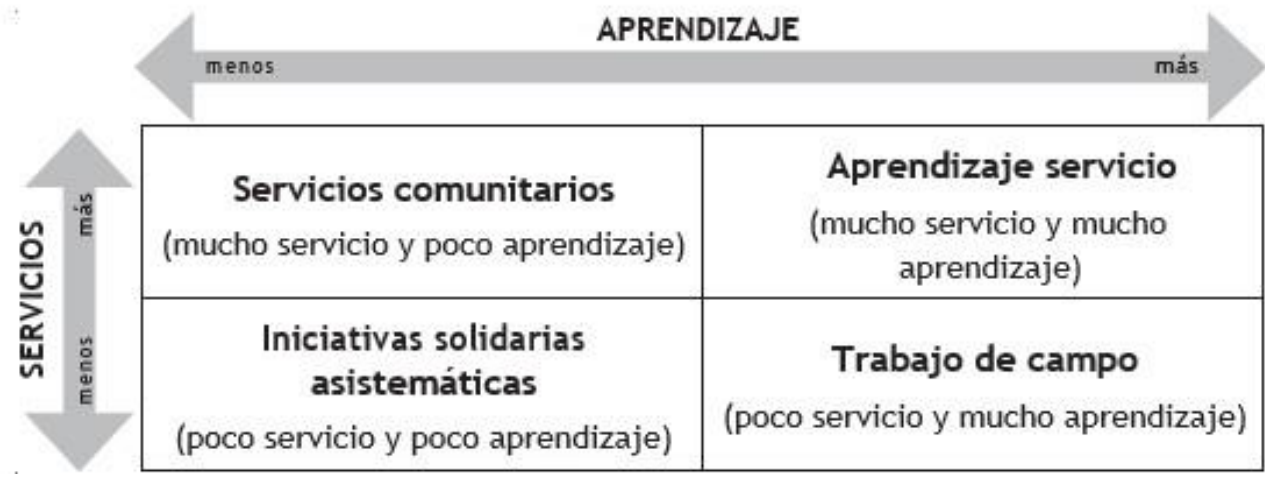

Cuadro 1. "Cuadrantes del aprendizaje servicio". Fuente: Puig et al. (2007).

Ya que la principal diferencia está en que el APS conecta los procesos de aprendizaje y de servicio a la comunidad en un proyecto bien articulado en el que se fomenta acciones formativas orientadas a promover el aprendizaje del alumnado, de forma significativa, útil y relevantes, a través de experiencias asociadas al servicio comunitario.

El APS, según Folgueiras et al. (2014), posibilita la realización de proyectos donde el alumnado puede involucrarse directamente, ocupando un papel relevante en todo el proceso, con aquellos a quienes ofrecen un servicio. El servicio que se ofrece es igual de importante que el aprendizaje de objetivos y deben adaptarse a las necesidades sociales.

Mayor y Rodríguez (2016) informan que el APS juega un papel relevante en los contextos educativos ampliados (escuela-comunidad), donde el profesorado y el estudiantado conformaran un grupo de investigación-acción para mejorar las necesidades de su entorno más cercano. 
Podemos destacar tres rasgos fundamentales descritos por Mayor y Rodríguez (2016) del APS: las personas implicadas presentarán una participación activa, se planificarán los objetivos curriculares destinados a conformar las acciones llevadas a cabo para dar un servicio y finalmente se dará un servicio a la comunidad para intentar mejorarla.

\section{Definiciones}

Existen diferentes puntos de vista para definir el APS. Nosotros nos centraremos en tres aproximaciones dadas por Puig et al. (2007); una primera aproximación al concepto de APS es observarlo como un programa de intervención a la comunidad para mejorar algunos aspectos de dicho entorno y desarrollar las capacidades cívicas del alumnado. Una segunda aproximación podría darse viendo el APS como una filosofía, pero sin dejar de ser un programa, en la que se intenta entender el crecimiento humano y elaborar un camino para crear comunidades más convivenciales y justas. Y finalmente entender el APS como un programa, una filosofía y una pedagogía, es decir, destacando la dinámica psicopedagógica a través de la cual el alumnado se educa; nuestra propuesta educativa se enmarca en este perspectiva.

Pero si lo vemos como práctica pedagógica según Mayor y Rodríguez (2016) el Aprendizaje y Servicio viene implementándose en el ámbito internacional desde los años 70.

Concretamente en el territorio español se inicia su difusión en los primeros años del siglo XXI en el País Vasco a través de la Fundación Zerbikas y en Cataluña en el Centro Promotor de Aprendizaje y Servicio.

Presentaremos a continuación algunas de las definiciones más notorias desde la perspectiva del ámbito internacional y desde la perspectiva del ámbito nacional (Cuadro 2).

\section{Ámbito Internacional}

\section{Ministerio de Educación chileno, 2007}

Eyler Y Gilers, 1999
El APS se traduce en proyectos pedagógicos de servicio comunitario, integrados en el currículum, en los que los estudiantes aplican, verifican y profundizan los aprendizajes aportando a la solución de un problema comunitario, permitiendo al estudiantado descubrir, aplicar y profundizar los conceptos disciplinarios en su vínculo con situaciones reales y la resolución de problemas concretos.

El APS es una forma de educación basada en la experiencia, en la que el aprendizaje se produce a través de un ciclo de acción y reflexión gracias al cual los estudiantes trabajan con otros compañeros en un proceso de aplicación de lo que han aprendido a los problemas de la comunidad y, al mismo tiempo, reflexionan sobre la experiencia de perseguir objetivos reales para la comunidad e incrementar su propia comprensión y destrezas, es decir, desarrollan de manera conexa las múltiples dimensiones humanas y cultivan la responsabilidad cívica y social. 


\begin{tabular}{|l|l|}
\hline \multirow{2}{*}{ Stanton, 1990} & $\begin{array}{l}\text { El APS es una forma de aprendizaje experiencial, una } \\
\text { expresión de valores -servicio a los demás- que determina } \\
\text { el objetivo, la naturaleza y el proceso de un intercambio } \\
\text { social y educativo entre los estudiantes y las personas a las } \\
\text { que ellos sirven, y entre los programas de educación y las } \\
\text { organizaciones comunitarias con las que ellos trabajan. }\end{array}$ \\
\hline Ámbito Nacional \\
\hline Puig Y Palos, 2007 & $\begin{array}{l}\text { Lo conciben como una propuesta educativa que combina } \\
\text { procesos de aprendizaje y de servicio a la comunidad en un } \\
\text { solo proyecto bien articulado, en el que los participantes se } \\
\text { forman al trabajar sobre necesidades reales del entorno con } \\
\text { el objetivo de mejorarlo }\end{array}$ \\
\hline $\begin{array}{l}\text { El APS es un método de enseñanza que enfatiza tanto el } \\
\text { aprendizaje académico que se desarrolla en el aula, como la } \\
\text { realización de un servicio voluntario a favor de las } \\
\text { necesidades detectadas en la comunidad próxima. }\end{array}$ \\
\hline 2007
\end{tabular}

El APS se define como un servicio solidario protagonizado por los estudiantes, destinado a atender necesidades reales y Tapia, 2005 efectivamente sentidas de una comunidad, planificado institucionalmente de forma integrada con el currículo, en función del aprendizaje de los estudiantes.

Cuadro 2. Definiciones de Aprendizaje y Servicio. Fuente: Francisco y Moliner (2010)

Todas las definiciones tanto del ámbito nacional como internacional coinciden en que es una práctica voluntaria cuyo fin es cubrir las necesidades de la comunidad. A continuación, enumeraremos la importancia de llevar a cabo este práctica educativa.

\section{Beneficios del Aprendizaje y Servicio}

¿Por qué el Aprendizaje y Servicio es tan importante? Debemos mencionar cuales son los principales benefactores de dicha metodología: los estudiantes, el profesorado, los equipos directivos y las entidades de la comunidad que colaboran para llevar a cabo el servicio; proporcionando así un clima favorable en los centros.

Según dicha clasificación, Puig et al. (2007) ha redactado cuales son los beneficios más significativos para cada uno de los sectores. En primer lugar, encontramos los/as alumnas de los centros educativos principales destinatarios de la metodología de APS. Hay que destacar los beneficios más claros, como son las mejoras académicas, sociales y emocionales, así como el desarrollo de múltiples destrezas intelectuales y personales. Por otra parte, el incremento de la responsabilidad cívica y de la participación activa en la comunidad. Y finalmente cabe mencionar la buena opinión que tienen los/s estudiantes de su participación en actividades de APS. 
En segundo lugar, encontraríamos el profesorado. Que según los resultados encontrados por Puig et al. (2007), llevar a cabo esta práctica mejora la opinión del profesorado sobre esta metodología y además aumenta su satisfacción como profesionales. Por otro lado, valoran el haber conseguido que el centro y la educación fuesen algo más relevante para sus alumnos, así como la colaboración con sus colegas y con las entidades sociales participantes.

En tercer lugar, hablemos de los equipos directivos de los centros, en especial del clima de convivencia y trabajo de las instituciones que dirigen. Según Puig et al. (2007) se observa una mejora evidente en la moral de los equipos docentes y del alumnado. Crece, por tanto, la disposición al trabajo, el buen humor, las buenas relaciones interpersonales, las ganas de innovar y la resistencia a las críticas. A su vez el alumnado y el centro perciben que la imagen de la escuela mejora y que la percepción que tienen de ella las demás instancias del entorno se hace más positiva.

En cuarto lugar, las entidades sociales que reciben el servicio a la comunidad también pueden lograr beneficios tangibles en la realización habitual de su cometido.

Y finalmente, tal y como resumen Puig et al. (2007) la puesta en marcha de procesos de APS puede ser beneficiosa para todos los implicados, pero ahora no tomados de modo individual sino en su conjunto. De este modo el APS, abre la posibilidad de que todas las instancias enumeradas puedan trabajar juntas, acordando ideas y coordinando planes de acción, para alcanzar unos objetivos comunes, que sean beneficiarios para todos.

Presentaremos ahora el Cuadro 3 donde encontramos a modo de síntesis, las aportaciones de Furco et al. (2002) sobre los beneficios de la participación en proyectos de Aprendizaje y Servicio:

\section{Impacto educativo del aprendizaje servicio}

\section{Académico y cognitivo}

O Aumento del rendimiento en pruebas estandarizadas.

- Mayor desarrollo de conocimientos conceptuales y competencias.

O Mayor asistencia, motivación respecto a la escuela y retención.

- Mejores notas promedio.

O Mayor habilidad para analizar y sintetizar información compleja. 


\begin{tabular}{|c|c|c|}
\hline \multirow{5}{*}{ Formación cívica } & & $\begin{array}{l}\text { Mayor comprensión de la política y de las actividades } \\
\text { gubernamentales. }\end{array}$ \\
\hline & 0 & $\begin{array}{l}\text { Mejor participación en la comunidad y en las } \\
\text { cuestiones públicas. }\end{array}$ \\
\hline & 0 & $\begin{array}{l}\text { Mejor ejercicio de la ciudadanía y de la } \\
\text { responsabilidad ciudadana. }\end{array}$ \\
\hline & 0 & $\begin{array}{l}\text { Mayor conciencia y comprensión de cuestiones } \\
\text { sociales. }\end{array}$ \\
\hline & 0 & Compromiso con el servicio comunitario. \\
\hline \multirow{4}{*}{$\begin{array}{l}\text { Vocacional y } \\
\text { profesional }\end{array}$} & 0 & $\begin{array}{l}\text { Ampliación de la conciencia y de las opciones } \\
\text { vocacionales. }\end{array}$ \\
\hline & 0 & Mejora de las competencias profesionales. \\
\hline & 0 & Mayor comprensión de la ética del trabajo. \\
\hline & 0 & Mejor preparación para el mundo del trabajo. \\
\hline \multirow[t]{3}{*}{ Ético y moral } & 0 & $\begin{array}{l}\text { Mayor exposición a nuevos puntos de vista y } \\
\text { perspectivas. }\end{array}$ \\
\hline & 0 & Cambios positivos en el juicio ético. \\
\hline & 0 & $\begin{array}{l}\text { Mayor habilidad para tomar decisiones } \\
\text { independientes respecto a cuestiones morales. }\end{array}$ \\
\hline \multirow[t]{5}{*}{ Personal } & O & $\begin{array}{l}\text { Ampliación de las cualidades y competencias para el } \\
\text { liderazgo. }\end{array}$ \\
\hline & 0 & Mayor autoestima. \\
\hline & 0 & Mayor conocimiento de sí mismo. \\
\hline & 0 & Mayor resiliencia. \\
\hline & 0 & Mayor eficacia personal. \\
\hline \multirow{4}{*}{ Social } & 0 & Mayor compañerismo entre estudiantes. \\
\hline & 0 & $\begin{array}{l}\text { Mayor habilidad para trabajar en equipos o para } \\
\text { trabajar con otros. }\end{array}$ \\
\hline & 0 & Capacidad para desechar los prejuicios. \\
\hline & 0 & Mejora de las conductas prosociales. \\
\hline
\end{tabular}

Cuadro 3. Impacto educativo del Aprendizaje del Servicio. Fuente: Furco (2004)

Por otro lado, los autores Martínez et al. (2013) recogieron una serie de investigaciones sobre Aprendizaje y Servicio en la Universidad y pudieron concluir a modo de síntesis que la participación activa en proyectos de APS permite desarrollar en el alumnado un aprendizaje más profundo y significativo. Por otro lado, se forman una serie de competencias profesionales y cívicas, habilidades personales, una mejora en la autoestima y un mayor 
conocimiento de uno mismo. Así como una mayor responsabilidad social y un alto grado de satisfacción. A modo ilustrativo presentaremos el cuadro con las investigaciones anteriormente mencionadas.

\begin{tabular}{|c|c|}
\hline ESTUDIOS & TEMAS INVESTIGADOS \\
\hline $\begin{array}{l}\text { Acquadro, Soro, } \\
\text { Biancetti and } \\
\text { Zanotta (2009) }\end{array}$ & $\begin{array}{l}\text { Necesidad de establecer vínculos entre universidad y voluntariado } \\
\text { en áreas de acción local. }\end{array}$ \\
\hline Annette (2005) & $\begin{array}{l}\text { El A-S vinculado a la educación moral y el fomento del deber y la } \\
\text { responsabilidad social. }\end{array}$ \\
\hline $\begin{array}{l}\text { Aramburuzabala } \\
(2012)\end{array}$ & $\begin{array}{l}\text { Impacto del A-S en el compromiso con la diversidad y la justicia } \\
\text { social }\end{array}$ \\
\hline $\begin{array}{l}\text { Bates, Drits, Allen y } \\
\text { McCandless } \\
(2009)\end{array}$ & $\begin{array}{l}\text { Desarrollo de competencias profesionales en estudiantes de } \\
\text { magisterio a través del A-S }\end{array}$ \\
\hline $\begin{array}{l}\text { Bednarz et al. } \\
(2008)\end{array}$ & $\begin{array}{l}\text { Estudio de casos de diferentes proyectos que siguen metodologías } \\
\text { participativas. }\end{array}$ \\
\hline $\begin{array}{l}\text { Bender y Jordaan } \\
(2007)\end{array}$ & Impacto del A-S en los programas de formación del profesorado \\
\hline $\begin{array}{l}\text { Carrington, } \quad \text { y } \\
\text { Saggers (2008) }\end{array}$ & $\begin{array}{l}\text { Desarrollo de competencias profesionales para la educación } \\
\text { inclusiva a través del A-S }\end{array}$ \\
\hline $\begin{array}{l}\text { Fernández y } \\
\text { Gaete }(2008)\end{array}$ & $\begin{array}{l}\text { Estudio Cualitativo sobre la satisfacción de los Socios } \\
\text { Comunitarios en los proyectos A-S }\end{array}$ \\
\hline $\begin{array}{l}\text { Folgueiras, Luna y } \\
\text { Puig (en } \\
\text { prensa) }\end{array}$ & $\begin{array}{l}\text { Grado de satisfacción de estudiantes universitarios que participan } \\
\text { en experiencias de A-S }\end{array}$ \\
\hline $\begin{array}{l}\text { Galambos and } \\
\text { Kozma }(2005)\end{array}$ & $\begin{array}{l}\text { Fenómenos que favorecen o dificultan la introducción de proyectos } \\
\text { de APS en la universidad }\end{array}$ \\
\hline
\end{tabular}




\begin{tabular}{|c|c|}
\hline Higgins (2009) & $\begin{array}{l}\text { Tesis doctoral en la que evalúa un proyecto de A-S de educación } \\
\text { para la ciudadanía global. }\end{array}$ \\
\hline $\begin{array}{l}\text { Holdsworth and } \\
\text { Quinn (2010) }\end{array}$ & Efectos que el voluntariado tiene en los estudiantes universitarios \\
\hline $\begin{array}{l}\text { Martínez-Odría } \\
\text { (2005) }\end{array}$ & $\begin{array}{l}\text { Tesis doctoral en la que se evalúa la incorporación curricular del } \\
\text { voluntariado a través del A-S }\end{array}$ \\
\hline Murphy (2010) & $\begin{array}{l}\text { Relación entre el A-s y la adquisición de competencias en } \\
\text { educación para la ciudadanía. }\end{array}$ \\
\hline Reinders (2010) & $\begin{array}{l}\text { Comparación de la enseñanza tradicional con otras que utilizan la } \\
\text { metodología de A-S }\end{array}$ \\
\hline $\begin{array}{l}\text { Root; Callahan y } \\
\text { Sepanski (2002) }\end{array}$ & $\begin{array}{l}\text { Revisión de estudios sobre A-S y desarrollo de competencias } \\
\text { docentes para la diversidad }\end{array}$ \\
\hline
\end{tabular}

Cuadro 4. Investigaciones de Aprendizaje y Servicio en la Universidad. Fuente: Martínez et al. (2013)

\section{Fases de la metodología de Aprendizaje y Servicio}

Para llevar a cabo un programa de Aprendizaje y Servicio es importante que se lleven a cabo los siguientes pasos:

$1^{\circ}$. Participación por parte del alumnado de forma auténtica. Para poder llevar a cabo un proyecto bajo la metodología de APS, es muy importante que los/as alumnos/as se involucren en él y que además se esfuercen por lograr las metas programadas. Para conseguir estos objetivos es fundamental crear un clima participativo, donde el alumnado se sienta motivado y con la confianza suficiente para participar activamente. Para ello, debemos desarrollar actividades que sean llamativas para los/as alumnos/as, así como hacer al alumnado consciente de su propio proceso de aprendizaje.

$2^{\circ}$. Diagnóstico exhaustivo de las necesidades reales de la comunidad. Conocer las necesidades de la comunidad no es una cuestión de simple curiosidad o información. Consiste en tomar conciencia sobre los problemas que son un obstáculo para el desarrollo grupal y se trata de hacerlo mediante un aprendizaje colectivo. Tal y como afirma Ibáñez (2008) dicho diagnóstico tiene un alto valor educativo en la medida en que todos aprendamos de todos y nos enriquezcamos con la experiencia y conocimiento. Las principales utilidades de hacer dicho diagnóstico sería: permitir identificar el desarrollo histórico de la comunidad, identificar los problemas y necesidades más relevantes y actuar con conocimiento pleno sobre esa realidad, contribuir a la motivación de la comunidad a identificar su realidad y a ganar niveles de conciencia sobre su papel transformador, constituye en un proceso de aprendizaje colectivo a partir del dialogo de saberes, promueve los niveles de comunicación y participación de la comunidad, permite desconcentrar poder y empoderar a la comunidad, genera autonomía 
para mejorar condiciones sociales y ayuda a cohesionar a la comunidad para actuar colectivamente

$3^{\circ}$. Colaboración con otros agentes del entorno. Las iniciativas de APS requieren de dicha colaboración para poder crear sinergias y enriquecer tal y como afirma Mendia (2016) al llamado "capital social" del centro, así como de las personas participantes.

$4^{\circ}$. Planificación intencional: Servicio a la comunidad y Objetivos curriculares. Debe ser un proyecto articulado explícitamente en el aprendizaje de contenidos curriculares. No es una simple acción "improvisada", anecdótica u ocasional, sino que es una acción educativa intencional, debidamente planificada.

$5^{\circ}$. Metodología: Investigación y Acción-participativa. Está basada en una actividad inclusiva que facilite la participación de todo el alumnado avanzando según dice Mendia (2016) en la superación de desigualdades e incidiendo positivamente en la equidad, empoderando al alumnado especialmente a aquel con especiales dificultades.

$6^{\circ}$. Poner en práctica el proyecto. Una vez, evaluadas todas las necesidades y habiendo articulado de forma correcta el proyecto basado en la metodología de APS, debe ejecutarse siguiendo todas las pautas propuestas.

$7^{\circ}$. Evaluación formativa. Difusión y celebración. La evaluación formativa mejora el aprendizaje del alumnado, del profesorado y de los procesos de enseñanza-aprendizaje, favoreciendo de este modo el aprendizaje autónomo.

Es decir, es necesario tal y como afirman Puig et al. (2011) que se lleve a cabo una serie de actividades complejas, en las que se organicen los objetivos y tareas que serán puestos en marcha y todo ello deberá integrarse en un proyecto bien articulado donde se ejecuten las distintas fases: realización de un diagnóstico de la realidad, desarrollo de un plan de acción, ejecutar la propuesta y finalmente la obtención de los resultados. Cuya finalidad principal será mejorar aquellos aspectos que haciendo uso de una mirada reflexiva y crítica obtendremos a través del compromiso cívico y la participación activa. Debemos tener en cuenta, según Puig et al. (2007) que para que sea posible la implantación de un programa de APS en los centros educativos es necesario el logro de un soporte organizativo y un reconocimiento legal.

Aun así, son diversos los centros educativos españoles que se embarcan en la gran aventura de implantar programas de Aprendizaje y Servicio a la comunidad. De hecho, en España se convocan cada año, desde el año 2015 cuando se inicio está propuesta, los premios de Aprendizaje y Servicio dónde cada centro presenta su proyecto de APS. Este año se han presentado 302 proyectos, del cual 36 pertenecen a Andalucía.

\section{SEGUNDA PARTE: PROPUESTA EDUCATIVA 5. CONTEXTUALIZACIÓN Y DESTINATARIOS}

En este TFG, hemos elaborado una propuesta educativa basada en la metodología de APS, que se ha llevado a cabo en el IES Alhamilla.

Los principales agentes de la implantación del programa son 7 alumnos/as de $2^{\circ}$ PMAR, que provienen principalmente de familias de etnia gitana. Por otro lado, los destinatarios del proyecto de APS que serán los 87 alumnos/as de $2^{\circ}$ ESO. 


\section{AGENTES}

Alumnado de $2^{\circ}$ PMAR

\section{DESTINATARIOS}

Alumnado de $2^{\circ}$ ESO

Además, se verán involucrada toda la comunidad educativa; es decir, el conjunto de profesores/as, los padres y las madres, el alumnado y el personal de administración y servicios.

\section{Objetivos}

El objetivo general de este proyecto es optimizar el desarrollo personal, conductual y rendimiento académico de los alumnos de $2^{\circ}$ de PMAR a través de una propuesta educativa basada en la metodología de Aprendizaje y Servicio.

Objetivos específicos:

1. Derivados del desarrollo personal; mejorar el autoconcepto y optimizar la capacidad de resiliencia.

2. Derivados del desarrollo conductual; reducir la tasa de absentismo escolar, así como mejorar la puntualidad del alumnado, y optimizar el uso de las habilidades sociales tales como: pedir y respetar el turno de palabra; uso de lenguaje apropiado y escucha activa en el aula.

3. Derivados del rendimiento académico; mejorar los resultados académicos del alumnado.

\section{Contenidos}

La temática utilizada para poder llevar a cabo este proyecto educativo es el uso inadecuado que los/as alumnos/as hacen de las redes sociales, concretamente de la aplicación de Instagram. Si observamos las alarmantes cifras del uso de las redes sociales recogidas en el Estudio Anual de Redes Sociales de IAB Spain, recopiladas por Acebes, Mozas, Montanera y Victori (2018); podemos ver que el $85 \%$ de la población son usuarios de las redes sociales, y entre estos, el $40 \%$ son estudiantes de secundaria. Concretamente Instagram está posicionada en $4^{\circ}$ lugar y destaca por ser de las tops Redes que más han aumentado la frecuencia de visita. Por otro lado, son la generación Z (personas de entre 16 y 23 años) las que hacen un mayor uso de las redes sociales.

El alumnado con el que trabajamos presenta edades comprendidas entre los 14 y 16 años, por lo cual son adolescentes potencialmente en riesgo de hacer un mal uso de esta aplicación, si no tienen la formación adecuada para ello. El principal problema es que la mayoría de los/as adolescentes desconocen los peligros de Instagram y las posibles consecuencias que puede ocasionar hacer un mal uso de dicha aplicación.

Tal y como viene redactado en el ABC, según Shirley Cramer, Directora Ejecutiva de "Royal Socitety for publchealth" (RSPH): "Las redes sociales han sido descritas como más adictivas que los cigarrillos y el alcohol, y ahora están tan arraigadas en las vidas de los jóvenes que ya no es posible ignorarlas cuando se habla de jóvenes" (Citado en el ABC, 2019). 


\section{METODOLOGÍA}

\section{Análisis de necesidades}

Antes de desarrollar las fases que llevaremos a cabo en este proyecto de APS, es imprescindible evaluar las necesidades más notorias de la comunidad educativa del centro.

En primer lugar, hemos utilizado como punto de línea base un análisis cualitativo a través de diversas entrevistas, cuyo objetivo es saber la opinión que los/as profesores/as que imparten clase en $2^{\circ}$ de PMAR tienen sobre los/as alumnos/as, en relación al comportamiento, la escucha activa y el lenguaje que utilizan en el aula.

Finalmente, hemos utilizado las notas de la primera evaluación para saber de dónde partimos en cuanto a su desarrollo académico.

Una vez definidas las principales necesidades de estos/as alumnos/as, se inicia la propuesta educativa.

\section{Fases}

\subsection{Evaluación inicial}

Aplicamos dos instrumentos de medida al alumnado de $2^{\circ}$ de PMAR. Por un lado, la Escala de Resiliencia y por otro, el cuestionario de Autoconcepto Dimensional, para comprobar la línea base de la que partimos en cuanto al desarrollo personal de este alumnado.

\subsection{Aprendizaje}

Una vez establecida la línea base, planificamos las distintas actividades que se pondrán en marcha para desarrollar la propuesta. Para ello, hemos realizado una serie de sesiones dónde se formará al alumnado de PMAR y se les darán los recursos y destrezas suficientes para llevar a cabo la fase de servicio.

Las actividades han de estar perfectamente organizadas, estableciendo el tiempo que se dedicará a cada actividad y teniendo en cuenta siempre los objetivos que queremos cumplir.

\subsection{Servicio}

Una vez que los/as alumnos/as han pasado el proceso de formación, comenzarán a difundir y comunicar a la comunidad educativa los conocimiento adquiridos durante la fase de aprendizaje.

\subsection{Evaluación final}

Evaluamos de nuevo al grupo de $2^{\circ}$ de PMAR, para comprobar si han mejorado su capacidad de resiliencia y su autoconcepto. Volveremos a entrevistar al profesorado, y comprobaremos cuales han sido los logros, tras esta propuesta.

\section{Sesiones}

\section{PREEVALUACIÓN: ANÁLISIS DE NECESIDADES}

\section{1ªsión: Análisis Cualitativo}

Objetivo: Detectar las necesidades del alumnado de PMAR, a través a las opiniones de sus profesores. 
Descripción: Se convoca una reunión, a la que deben asistir los/as profesores/as que imparten clase en $2^{\circ}$ de PMAR. Una vez que estén todos/as reunidos/as, haremos una serie de preguntas que deberán responder uno a uno, tales como: ¿Qué tipo de lenguaje utilizan los/las alumnos/as en clase? ¿Respetan las intervenciones de sus compañeros y profesores? ¿Piden el turno de palabra para hablar en clase?

Recursos: Papel, bolígrafos, listado del profesorado de $2^{\circ}$ de PMAR para llevar un registro de todos los que han asistido a la reunión, listado de los alumnos de PMAR para saber más detalladamente de cada uno de ellos.

Lugar: Sala de Profesores

Duración: 1 hora y media.

\section{$2^{\circ}$ Sesión: Análisis Cuantitativo}

Objetivo: Detectar las necesidades de PMAR en cuanto a su desarrollo académico.

Descripción: Reunir de nuevo, al profesorado y analizar cuáles han sido las notas del alumnado en la $1^{\text {a }}$ Evaluación.

Recursos: Papel, bolígrafos y listado de las notas del alumnado en la $1^{\text {a }}$ Evaluación.

Lugar: Sala de Profesores

Duración: 1 hora

$1^{a}$ Fase: EVALUACIÓN

$\underline{1^{a} \text { Sesión: Evaluación inicial Resiliencia }}$

Objetivo: Evaluación inicial a cada alumno/a, a través de la Escala de Resiliencia de Connor y Davidson, CD-RISC.

Descripción: Comenzamos introduciendo el concepto de resiliencia tal y como afirman Rodríguez, Ramos y Ros (2015), como el afrontamiento adecuado de las tareas evolutivas propias de una determinada etapa de desarrollo a pesar de la adversidad. Además, según Serrano, Garrido, Notario, Bartolomé, Solera y Martínez-Vizcaino (2012) la resiliencia es considerada un importante factor de salud mental y además está relacionada de forma positiva con la autoeficacia, el autocontrol y la competencia personal. Esta prueba fue creada en el año 2003 y consta de dos versiones fundamentales, una de 25 ítems y la otra de 10 (Connor Y Davidson, 2003). En nuestro caso hemos utilizado la escala de 25 ítems. Es una escala tipo Likert que oscila entre $0=$ nada de acuerdo, a $4=$ totalmente de acuerdo. Donde la puntuación máxima es de 100, reflejando las puntuaciones más altas mayor resiliencia.

Se reúne al alumnado en clase y se le aplica el cuestionario, explicando uno a uno los ítems y las posibles alternativas de respuesta.

Recursos: Cuestionario de Resiliencia, bolígrafos, pizarra y tiza para aclarar algunas dudas que puedan surgir.

Lugar: Aula.

Duración: 1 hora. 


\section{$\underline{2^{\circ} \text { Sesión: Evaluación inicial autoconcepto }}$}

Objetivo: Evaluación inicial a cada alumno/a, a través del cuestionario de Autoconcepto Dimensional (AUDIM) de Fernández-Zabala, Goñi, Rodríguez-Fernández y Goñi (2015).

Descripción: Entendemos el autoconcepto según Rodríguez et al. (2015) como un indicador del bienestar personal que influye positivamente en las conductas ajustadas y adaptativas en la adolescencia. El cuestionario posee un formato de respuesta en escala Likert de cinco grados que oscila desde 1 = falso a 5 = verdadero y consta de 33 ítems. Consta de 11 escalas que evalúan dimensiones del autoconcepto, además de una escala general para la medida del autoconcepto, que se define como la autopercepción global de la persona.

El procedimiento será igual a como hicimos la aplicación del cuestionario de Resiliencia.

Recursos: Cuestionario de Resiliencia, bolígrafos, pizarra y tiza para aclarar algunas dudas que puedan surgir.

Lugar: Aula.

Duración: 1 hora.

\section{$2^{\circ}$ Fase: APRENDIZAJE DE LOS AGENTES}

\section{$\underline{3^{a} \text { Sesión: Consensuando normas }}$}

Objetivo: Presentación del concurso que será llevado a cabo durante toda la propuesta educativa.

Descripción: Explicaremos a los/as alumnos/as que vamos a realiza con ellos un concurso, cuya temática principal gira en torno a Instagram. El nombre del concurso es: "Instagramers en acción". Debemos explicarles que este concurso consiste en cumplir una serie de normas durante todas las sesiones que durará el concurso. Las normas propuestas son las siguientes:

1. Venir a clase de forma puntual. El registro se llevará a cabo pasando lista a las 9:15 h. al comienzo de la clase. Al alumno/a que cumpla este requisito se le otorgará el valor de 10 puntos.

2. Respeto durante las intervenciones. El alumno/a que interrumpa a las profesoras o a alguno de sus compañeros/as mientras intervienen durante la sesión, se les quitará 1 punto. Pueden alcanzar hasta un máximo de 10 puntos.

3. Pedir el turno de palabra. El alumnado que no levante la mano para hablar durante la sesión será penalizado con 1 punto menos. Pudiendo alcanzar hasta un máximo de 10 puntos.

4. Hacer uso de un lenguaje apropiado. Durante las clases se hablará con respeto tanto al profesorado como a los compañeros/as de clase. Podrán obtener como máximo 10 puntos, restándole 1 punto si no utilizan el lenguaje adecuado.

5. Participación activa. El alumnado deberá tener predisposición y mostrar interés durante las actividades llevadas a cabo en cada sesión. Si este objetivo se cumple se les dará 10 puntos.

Si son capaces de respetar estas cinco normas, podrán conseguir hasta un máximo de 50 puntos en cada sesión docente. Para conseguir una mayor motivación para el alumnado, cada semana se mostrará un ranking en el que se verá plasmado el progreso de cada alumno/a y los 
puntos obtenidos en cada sesión. Durante todas las sesiones se llevará a cabo un registro de cada alumno, con las normas que han cumplido y la puntuación final obtenida.

Al final de todas las sesiones, los dos mejores alumnos/as de la clase podrán clasificarse en $1^{\circ}$ y $2^{\circ}$ puesto, pudiendo ganar un premio. El premio al que podrán optar será una taza con una frase motivadora y la oportunidad de mostrar los conocimientos adquiridos durante el concurso en la Fundación Secretariado Gitano.

Recursos: Proyector y Power Point explicativo con las normas del concurso.

Lugar: Aula

Duración: 1 hora.

\section{$\underline{4^{a} \text { Sesión: Búsqueda de "Likes" en Instagram }}$}

Objetivo: Mostrar la espiral del postureo en las redes sociales para posteriormente realizar un debate sobre el vídeo. Trabajando así la capacidad que tiene el alumnado de poner en orden las ideas aprendidas durante la sesión y la capacidad de organizarse entre ellos mismos para realizar el debate.

Descripción: Antes de comenzar la sesión debe proyectarse el Power Point, dónde se explicará las actividades que se llevarán a cabo en la sesión. A continuación, se mostrará al alumnado este cortometraje https://youtu.be/PjndafVyyYY con una duración de 2:36 minutos. En este vídeo se muestra con todo lujo de detalles la espiral del postureo en las redes sociales. El contenido del vídeo presenta la historia de un hombre cuya vida gira en torno a los "likes" de una red social, donde realiza publicaciones de frases o fotos de cosas que en realidad no hace como por ejemplo publicar una foto haciendo deporte cuando en realidad ha ido en coche. Cada vez se obsesiona por tener más likes y finalmente se da cuenta de que ha echado parte de su vida a perder y lo borra todo. Una vez finalizado el vídeo, es recomendable que se explique a la perfección el mensaje que intenta transmitir y que se aclaren las posibles dudas que puedan surgir.

A continuación, se llevará a cabo el debate del vídeo. Para ello debe dividirse el alumnado formando dos equipos. Uno de los equipos se encargará de redactar en un folio los aspectos positivos de Instagram y el otro equipo los aspectos negativos.

Una vez finalizada esa parte, deben dividirse las mesas y las sillas de forma, que ambos equipos se encuentren unos delante de otros. A continuación, se seleccionará un alumno que será el que se encargue de moderar todo el debate, la primera pregunta que lanzará será: "Primer equipo, ¿Cuáles creéis que podría ser un aspecto positivo de Instagram?” A raíz de esta pregunta, el primer equipo argumentará el primer aspecto positivo que tenía ya redactado, y el equipo contrario tendrá que debatirlo y además añadir a su defensa algún aspecto negativo que haya redactado en el folio anterior.

Recursos: Proyector, vídeo, altavoces, ordenador, Power Point explicativo de la sesión, folios, bolígrafos y hoja de registro de los alumnos.

Lugar: Aula

Duración: 1 hora 


\section{$\underline{5^{\circ} \text { Sesión y } 6^{\circ} \text { Sesión: Mural de Instagram }}$}

Objetivo: Realizar un mural que ilustre los 7 aspectos positivos y los 7 aspectos negativos de Instagram.

Descripción: Antes de iniciar la actividad, debemos mostrarles en el Power Point del concurso el ranking de la sesión anterior, dónde se mostrará la posición que ha ocupado cada alumno y la puntuación final. Todo ello en función de si han cumplido o no las normas. A continuación, se reparte una cartulina, dos folios que muestran 14 capturas de pantalla de la aplicación de Instagram y un folio con 7 aspectos positivos tales como: 1) La rapidez de la información, 2) Mayor acceso a perfiles de interés, 3) Facilidad para retomar el contacto, 4) Permiten acceder a todo tipo de contenido, 5) Facilidad para la autopromoción, 6) Fuente de entretenimiento, 7) Venta online y 7 aspectos negativos del uso de dicha aplicación: 1) Exhibicionismo selectivo, 2) Fragilidad de nuestra privacidad, 3) Viralidad de rumores sin contrastar, 4) Exceso de vanidad, 5) Los errores pueden salir muy caros, 6) Es fácil perder el tiempo, 7) Exceso de publicidad.

Los/as alumnos/as deben emparejar cada imagen con la frase correspondiente. A continuación, deben dividir la cartulina en dos y en un lado pegar las 7 imágenes con las 7 frases que describen los aspectos positivos y en el otro lado de la cartulina hacer lo mismo con los aspectos negativos. Creando así un mural ilustrativo.

Recursos: Cartulina, Pegamento, Colores, Recortes de las imágenes y las frases, Proyector, Power Point explicativo de la sesión y del primer ranking y hoja de registro de los alumnos.

Lugar: Aula de Plástica Duración: 2 horas.

\section{$\underline{7^{\circ} \text { Sesión: Ciberbullying }}$}

Objetivo: Explicación del ciberbullying y de los peligros que puede ocasionar.

Descripción: Antes de iniciar la actividad, debemos mostrarles en el Power Point del concurso, el ranking de la sesión anterior dónde se mostrará la posición que ha ocupado cada alumno y la puntuación final. Todo ello en función de si han cumplido o no las normas. A continuación, se explicará que la temática de la clase girará en torno al ciberbullying. Para aclarar las posibles dudas sobre, qué es el ciberbullying se proyectará el siguiente vídeo en clase https://www.youtube.com/watch?v=G8iciqvXnmk, presenta una duración de 6:37 minutos.

El contenido del vídeo muestra la historia de una niña que recibe ciberacoso a través de internet, la protagonista desconoce los peligros de las redes sociales y no se percata de la posibilidad de que haya perfiles falsos. En esta historia un hombre de 45 años se hace pasar por su novio y le pide que le envíe fotos comprometidas de ella. La niña cede a las peticiones y el acosador revela su identidad y le pide una elevada cantidad de dinero si no quiere que muestre a todo el mundo sus fotos.

Tras ver el vídeo se aclararán las posibles dudas que puedan surgir. Para concienciar aún más al alumnado sobre este problema se proyectará en el proyector 5 noticias del periódico del $\mathrm{ABC}$ sobre distintos casos reales de ciberbullying en España: https://www.abc.es/familia/educacion/abci-victimas-bullying-pueden-sufrirenfermedadesmentales-201901300301_noticia.html https://www.abc.es/espana/castillaleon/abci-detenidomenor-miranda-extorsionar-y-amenazar-otro-traves-internet201811071425_noticia.html https://www.abc.es/sociedad/abci-agreden-nina-12-anos-tarifa-ygraban-video- 
201709131100_noticia.html https://www.abc.es/espana/aragon/abci-detenido-cuartaciberacosador-aboco-suicidio-menor201703171323_noticia.html

https://www.abc.es/familia/educacion/abci-como-protocolo-actuacion-caso-acosoescolar201701160228_noticia.html.

Para finalizar la actividad, se le pedirá a cada alumno/a que redacte en medio folio algún caso de acoso en las redes sociales que hayan presenciado. Una vez escrito deben meterlo de forma anónima en un sobre, que les será proporcionado, con el fin de concienciarlos sobre este tema que nos afecta a todos y que además es una realidad que ocurre cada vez con más frecuencia.

Recursos: Proyector, altavoces, folios, bolígrafos, sobres, vídeo, noticias del ABC, Power Point explicativo de la sesión y del ranking de la sesión anterior, hoja de registro de los alumnos.

Lugar: Aula.

Duración: 1 hora.

\section{$\underline{8^{\circ} \text { Sesión: Postureo en Instagram }}$}

Objetivo: Concienciar sobre el postureo de Instagram y los peligros que ello conlleva. Descripción: Al igual que en las sesiones anteriores se mostrará el PPT del concurso, con el ranking semanal y las actividades propuestas. La temática esta sesión gira en torno al postureo, según la RAE (Real Academia Española) el postureo es la "actitud artificiosa e impostada que se adopta por conveniencia o presunción”, en las redes sociales. Para poder trabajar sobre ello, la primera actividad consistirá en pedirle a los/as alumnos/as que saquen sus móviles y que busquen en Instagram famosos/as a los que ellos seguían. Una vez hecha la búsqueda, se proyectarán los perfiles que haya buscado y comentaremos los aspectos negativos de esta actitud. A continuación, expondremos las consecuencias que pueden darse en los/as adolescentes que se obsesionan por querer aparentar una vida totalmente contraria a la suya, pudiendo provocar trastornos de ansiedad o depresión.

Recursos: Teléfonos móviles, proyector, PPT explicativo de la sesión y ranking semanal, hoja de registro.

Lugar: Aula

Duración: 1 hora

\section{Sesión: Kahoot de los contenidos aprendidos}

Objetivo: Asentar los conocimientos aprendidos durante las sesiones y preparación de la fase de servicio.

Descripción: Al igual que en las sesiones anteriores se mostrará el PPT del concurso, con el ranking semanal y la actividad propuesta. Para asentar todos los contenidos dados durante la fase de aprendizaje, realizaremos un Kahoot. El enlace del Kahoot es el siguiente https://create.kahoot.it/kahoots/my-kahoots.

A continuación, se le explicará al alumnado que todos los conocimientos aprendidos deberán enseñárselo al resto de sus compañeros de $2^{\circ} \mathrm{ESO}$ en las próximas sesiones.

Recursos: Teléfonos móviles, proyector, PPT explicativo de la sesión y ranking semanal, hoja de registro. 
Lugar: Aula de Informática.

Duración: 30 minutos.

\section{$3^{a}$ Fase: SERVICIO A LOS DESTINATARIOS}

Las sesiones propuestas tendrán la misma dinámica y se realizarán las mismas actividades. Esto es debido a que los/as alumnas de $2^{\circ} \mathrm{PMAR}$ pertenecen a clases de $2^{\circ} \mathrm{ESO}$ diferentes, por lo tanto, por una cuestión de horario y de organización por parte del alumnado y del profesorado, estas sesiones serán impartidas en horario de tutoría a los cursos de $2^{\circ} \mathrm{ESO} \mathrm{A}, 2^{\circ}$ ESO B Y $2^{\circ}$ ESO C.

\section{$\underline{10^{\mathrm{a}}, 11^{\mathrm{a}} \text { y } 12^{\mathrm{a}} \text { Sesión }}$}

Objetivo: Enseñar al alumnado de $2^{\circ} \mathrm{ESO}$, los siete aspectos positivos y negativos de Instagram.

Descripción: Los/as alumnos/as de PMAR, se presentan al resto de sus compañeros y les explican que, durante esta sesión, van a explicarles los aspectos positivos y negativos de Instagram. Para poder hacer más divertida la sesión los alumnos de PMAR serán los capitanes de cinco grupos que se formarán en la clase de $2^{\circ}$ ESO. Para ello, se procederá a dividir las mesas y las sillas de forma que queden los cinco grupos en una zona diferente del aula.

Cada capitán del grupo de alumnos de $2^{\circ}$ ESO les lanzará la siguiente pregunta: ¿Conocéis 7 aspectos negativos y 7 aspectos positivos de Instagram? A raíz de esta pregunta se formará una lluvia de ideas y el capitán del grupo deberá escribir todas las ideas en un folio.

Una vez hecho esto, los capitanes de los grupos mostrarán al resto de sus compañeros el mural que realizaron y explicarán con todo lujo de detalle lo 7 aspectos negativos y positivos de la aplicación de Instagram.

Recursos: Mural de Instagram, folios, bolígrafos, pizarra y tizas para aclarar dudas.

Lugar: Aulas de $2^{\circ}$ ESO A, B Y C.

Duración: 3 horas.

\section{$\underline{13^{\mathrm{a}}, 14^{\mathrm{a}}, 15^{\mathrm{a}} \text { Sesión: Ciberbullying }}$}

Objetivo: Trabajar el ciberbullying a través de una historia basada en un hecho real.

Descripción: Los/as alumnos/as de PMAR comienzan la sesión lanzando la siguiente pregunta a la clase: “¿Qué es el ciberbullying?”. A continuación, explicaran el concepto de ciberbullying y las conductas típicas de este concepto tales como: amenazar, robar contraseñas, suplantar identidades, blog y fotologs, uso o creación de webs con contenidos ofensivos, enviar fotografías o vídeos por WhatsApp o Instagram, encuestas de Internet, insultos a través de juegos multiusuario o enviar programas basura.

Tras estas explicaciones se expondrá una historia basada en un hecho real, pero con nombres ficticios para finalmente debatir entre todos las siguientes preguntas: ¿Por qué hay chicas que se meten con otras chicas? ¿En qué se equivocó Samanta? Imaginad un buen final, donde TODOS se acaben sintiendo bien. ¿Cómo sería? ¿Pensáis que es una historia muy extraña? ¿Sucede a menudo? ¿Conocéis personas que hagan cosas así? ¿Qué significa la frase "Vive y deja vivir"?

Recursos: Power Point, pizarra, tizas. 
Lugar: Aulas de $2^{\circ}$ ESO A, B Y C.

Duración: 4 horas y media.

$\underline{16^{\mathrm{a}}, 17^{\mathrm{a}}, 18^{\mathrm{a}} \text { Sesión: Proyectar los conocimientos adquiridos a las familias }}$

Objetivo: Mostrar a todos los familiares de los alumnos de $2^{\circ}$ ESO y $2^{\circ}$ PMAR los contenidos adquiridos por los alumnos de PMAR.

Descripción: Se convoca a todos los familiares de los alumnos de $2^{\circ}$ ESO A, $2^{\circ}$ ESO B, $2^{\circ}$ ESO C y $2^{\circ}$ PMAR a una reunión. Los protagonistas de dicha reunión serán los alumnos de $2^{\circ}$ de PMAR que, con la ayuda de un PPT, explicarán a todos los familiares los contenidos adquiridos durante las sesiones. Por otro lado, se les sugerirá a todos los familiares, que tengan un cierto control sobre el uso que sus hijos hacían de la aplicación de Instagram. Ya que en muchas ocasiones un caso de ciberbullying pude pasar de desapercibido.

Recursos: Power Point Lugar:

Salón de actos.

Duración: 2 horas.

- Proyección del servicio

Objetivo: Colaborar con la Asociación del Secretariado Gitano de Almería, para trabajar junto a ellos los peligros de usar inadecuadamente la aplicación de Instagram.

Descripción: Los alumnos de PMAR ganadores del concurso, colaborarán con esta asociación para ir por los distintos IES de Almería enseñando a los distintos alumnos de la ESO sobre los peligros que puede ocasionar el Instagram, si se hace un mal uso de esta aplicación.

Recursos: Mural ilustrativo y PPT, más los recursos aportados por esta asociación.

Lugar: Distintos institutos de Almería.

Duración: Proyecto educativo de duración variable.

$4^{\circ}$ Fase: EVALUACION

$19^{a}$ Sesión: Evaluación final de Resiliencia

Objetivo: Evaluación inicial a cada alumno/a, a través de la Escala de Resiliencia de Connor y Davidson, CD-RISC.

Descripción: Se reúne al alumnado en clase y se le aplica el cuestionario, explicando uno a uno los ítems y las posibles alternativas de respuesta.

Recursos: Cuestionario de Resiliencia, bolígrafos, pizarra y tiza para aclarar algunas dudas que puedan surgir. Lugar: Aula

Duración: 1 hora

\section{$\underline{20^{a} \text { Sesión: Evaluación final de Autoconcepto y entrega de premios }}$}

Objetivo: Evaluación inicial a cada alumno/a, a través del cuestionario de Autoconcepto Dimensional (AUDIM) de Fernández-Zabala, Goñi, Rodríguez-Fernández y Goñi (2015).

Descripción: Se reúne al alumnado en clase y se le aplica el cuestionario, explicando uno a uno los ítems y las posibles alternativas de respuesta. Para finalizar la sesión mostramos el 
PPT con el ranking final y hacemos entrega de las tazas y les revelamos que tienen la oportunidad de mostrar los conocimientos adquiridos durante el concurso en la Fundación Secretariado Gitano.

Recursos: Cuestionario de Autoconcepto, bolígrafos, pizarra y tiza para aclarar algunas dudas que puedan surgir, PPT con el ranking final.

Lugar: Aula

Duración: 1 hora y media.

\section{$21^{\text {a }}$ Sesión: Opinión cualitativa de los estudiantes}

Objetivo: Recoger información sobre la opinión que los profesores tienen sobre los estudiantes de PMAR tras haber finalizado la propuesta educativa basada en la metodología APS.

Descripción: Se convoca una última sesión con todo el profesorado de $2^{\circ}$ de PMAR. Donde recogeremos las opiniones que de forma cualitativa nos darán el profesorado al responder a las siguientes preguntas: ¿Han mejorado su comportamiento durante las clases? ¿Utilizan un lenguaje adecuado? ¿Han dejado de insultar y decir palabrotas? ¿Respetan los turnos de palabra? ¿Realizan las intervenciones de clase levantando la mano? ¿Respetan a sus compañeros? ¿Os respetan a vosotros durante las clases? ¿Participan de forma activa? A continuación, les pediremos que nos enseñen las notas de la tercera evaluación para comprobar si ha habido mejora en cuanto a su desarrollo académico. Y, finalmente, les mostraremos a todo el profesorado el registro que hemos estado llevando durante todo el concurso, donde se ha recogido una serie de aspectos tales como: 1) Venir a clase de forma puntual, 2) Respeto durante las intervenciones, 3) Pedir el turno de palabra, 4) Hacer uso de un lenguaje apropiado y 5) Participación activa por parte del alumnado.

Recursos: Hoja de registro del alumnado, notas de la $3^{\text {a }}$ Evaluación, bolígrafos y folios.

Lugar: Sala de Profesores.

Duración: 2 horas.

\section{CRONOGRAMA}

A continuación, se muestra un cronograma, el cual servirá como desarrollo de la temporalización del proyecto de innovación que vamos a llevar a cabo. En el aparecen las fases de la metodología de Aprendizaje y Servicio y las sesiones por semana que se realizaran durante un periodo de seis meses. La duración de cada sesión será de 1 hora a la semana, en horario de tutoría, excepto en algunas sesiones, en las que el tiempo empleado será diferente y por lo tanto será especificado en cada apartado. 


\begin{tabular}{|c|c|c|c|c|c|c|c|c|c|c|c|c|c|c|c|c|c|c|c|c|c|c|c|c|}
\hline \multirow{2}{*}{ Sesiones por semana } & \multicolumn{4}{|c|}{ Enero } & \multicolumn{4}{|c|}{ Febrero } & \multicolumn{4}{|c|}{ Marzo } & \multicolumn{4}{|c|}{ Abril } & \multicolumn{4}{|c|}{ Mayo } & \multicolumn{4}{|c|}{ Junio } \\
\hline & 1 & 2 & 3 & 4 & 1 & 2 & 3 & 4 & 1 & 2 & 3 & 4 & 1 & 2 & 3 & 4 & 1 & 2 & 3 & 4 & 1 & 2 & 3 & 4 \\
\hline \multicolumn{25}{|l|}{ PREEVALUACIÓN: } \\
\hline \multicolumn{25}{|l|}{ 1a Fase: EVALUACIÓN } \\
\hline \multicolumn{25}{|l|}{ 20 Fase: APRENDIZAJE } \\
\hline \multicolumn{25}{|l|}{ 3a Fase: SERVICIO } \\
\hline $\begin{array}{l}\text { 4 Fase: EVALUACIÓN } \\
\text { FINAL }\end{array}$ & & & & & & & & & & & & & & & & & & & & & & & & \\
\hline
\end{tabular}

\section{RESULTADOS ESPERADOS}

He tenido la oportunidad de llevar a la práctica parte de este TFG, concretamente la parte de preevaluación, la fase de Aprendizaje y parte de la fase de evaluación final. Pero por cuestión de tiempo y organización con el IES. Alhamilla, no he podido terminar con éxito esta propuesta educativa basada en la metodología de APS. Aún así expondré algunos de los logros obtenidos en cuanto a los objetivos propuestos y las mejoras que he podido observar en el alumnado.

Respecto al primer objetivo en el que se proponía "mejorar el autoconcepto y optimizar la capacidad de resiliencia". Sólo pudimos aplicar el cuestionario de Resiliencia. Pero si se aplicasen ambos existiría una correlación positiva. Ya que, según Rodríguez et al. (2015) existe una asociación positiva entre la resiliencia y el autoconcepto-autoestima y además el autoconcepto de los adolescentes predice de forma positiva la conducta resiliente. Además, estos mismos autores Rodríguez et al. (2015) apuestan por intervenciones preventivas que sirvan para equipar a los jóvenes con aquellas habilidades necesarias para la gestión adecuada de posibles problemas o dificultades. De manera específica, se ha demostrado la efectividad de programas de promoción de la resiliencia en el contexto escolar que impulsan el empoderamiento y las autopercepciones positivas considerando como factores ambientales las relaciones sociales.

Se observó que tras los resultados obtenidos en el cuestionario de Resiliencia que fue aplicado en la fase de evaluación inicial y los resultados obtenidos tras aplicar el mismo cuestionario en la fase de evaluación final, hubo una mejora en la capacidad de resiliencia de los alumnos. El segundo objetivo proponía "Reducir la tasa de absentismo escolar, así como mejorar la puntualidad del alumnado, y optimizar el uso de las habilidades sociales tales como: pedir y respetar el turno de palabra; uso de lenguaje apropiado y escucha activa en el aula. Este objetivo fue evaluado con el registro semanal del alumnado, donde se recogía si el alumno había respetado o no las normas del concurso de "Instagramers en acción". Siendo las normas: 1. Venir a clase de forma puntual, 2. Respeto durante las intervenciones, 3. Pedir el turno de palabra, 4. Hacer uso de un lenguaje apropiado, 5. Participación activa.

Comprobamos que antes de iniciar este proyecto, había una tasa de absentismo de entre dos y tres alumnos por día. Al principio durante el registro, pudimos notar como a los/as alumnas les costaba bastante respetar y pedir los turnos de palabra y hablar de manera apropiada. Sin 
embargo, gracias a la creación del concurso y a la motivación de obtener el máximo número de puntos para poder optar al premio final, conforme avanzaban las sesiones se iba viendo un resultado muy favorable en los/as alumnos/as. El último objetivo perseguido pretendía "mejorar los resultados académicos del alumnado". Este último objetivo no llegamos a comprobarlo ya que no pudimos tener una reunión final con los profesores de $2^{\circ}$ de PMAR. Finalmente, propusimos como premio final participar en la asociación del Secretariado Gitano de Almería. Elegimos esta asociación ya que la mayoría del alumnado de $2^{\circ}$ de PMAR, vienen de familias gitanas y esta oportunidad podría empoderar aun más a los/as alumnos/as; ya que se sentirían realizados tanto a nivel académico como a nivel personal.

A modo de conclusión, podemos decir que todos los que han tenido la oportunidad de participar en parte de este proyecto han experimentado, algunos de los beneficios de la metodología de APS. Tal y como afirma Batlle (2011), hacer un servicio a la comunidad, ayudar a los otros, es uno de los métodos más eficaces de aprendizaje, porque los/as estudiantes encuentran sentido a lo que estudian cuando aplican sus conocimientos y habilidades en una práctica solidaria.

\section{REFERENCIAS BIBLIOGRÁFICAS}

ABC (2017). Instagram, la red social que más afecta a la salud mental de los jóvenes. Recuperado de https://www.abc.es/tecnologia/redes/abci-instagram-social-masafecta-saludmental-jovenes-201705222045_noticia.html

Acebes, B., Mozas, C., Montanera, R. y Victori, J. (2018). Estudio Anual de Redes Sociales. Recuperado de https://iabspain.es/estudio/estudio-anual-de-redes-sociales-2018/

Barrio, P. (2006). Calidad, equidad e indicadores en el sistema educativo español. Pulso. Revista de educación, (29), 43-58.

Batlle, R. (2011). Voluntariado y aprendizaje-servicio. Revista do Seminario Galego de Educación para la Paz, 3-6.

Connor, M. y Davidson, R. (2003). Development of a new resilience scale: The ConnorDavidson resilience scale (CD-RISC). Depression and anxiety, 18(2), 76-82.

Corujo, M. D. C., Méndez, S., y Rodríguez, A. M. (2018). Valoración de los Programas de Mejora del Aprendizaje y del Rendimiento desde la visión de sus protagonistas en cuatro poblaciones de la provincia de Sevilla. Tendencias Pedagógicas, (32), 31-48.

Fernández, A., Goñi, E., Rodríguez, A. y Goñi A. (2015). Un nuevo cuestionario en castellano con escalas de las dimensiones del autoconcepto. Revista Mexicana de Psicología, (2), 149159.

Folgueiras, P., Luna, E. y Puig, G. (2014). El Aprendizaje y servicio en educación secundaria. Revista Iberoamericana de Educación, (20), 127-145.

Francisco, A. y Moliner, L. (2010). El Aprendizaje Servicio en la Universidad: una estrategia en la formación de ciudadanía crítica. Revista Electrónica Interuniversitaria de Formación del Profesorado, (4), 69-77. 
Furco, A., y Billig, S. (2002). Service learning: The essence of the pedagogy. Information age publishing, (34), 201-245.

Ibáñez, C. (2008). Participación comunitaria y diagnóstico de necesidades. Recuperado de https://www.madrimasd.org/blogs/salud_publica/2008/11/17/107090

Martínez, B., Martínez, I., Alonso, I., y Geruzaga, M. (2013). El aprendizaje-servicio, una oportunidad para avanzar en la innovación educativa dentro de la Universidad del País Vasco. Tendencias Pedagógicas, (21), 99-117.

Mayor, D., Rodríguez, D. (2016). Aprendizaje-Servicio y práctica docente: una relación para el cambio educativo. Revista de Investigación Educativa. (34), 535-552.

Mendia, R. (2016). El Aprendizaje-Servicio: Una metodología para la innovación educativa. Revista Convives. Recuperado de http://www.zerbikas.es/zerbika/

Ministerio de Educación y Formación Profesional (2018). Informe 2018 sobre el sistema educativo. Recuperado de http://www.educacionyfp.gob.es/educacion/mc/cee/publicaciones/informes-delsistemaeducativo/informe-2018.htm

Puig, M., Batlle, R., Bosch, C., y Palos, J. (2007). Aprendizaje servicio. Educar para la ciudadanía. Barcelona, España, Octaedro.

Puig, J. M., Gijón, M., Martín, X., y Rubio, L. (2011). Aprendizaje-servicio y Educación para la Ciudadanía. Revista de Educación. 45-67.

Red Española de Aprendizaje-Servicio (2018). Memoria de los premios AprendizajeServicio. Recuperado de https://aprendizajeservicio.net/premios-aps-2018/

Rodríguez, A., Ramos, E., Ros, I. y Fernández, A. (2015). Relaciones de la resiliencia con el autoconcepto y el apoyo social percibido en una muestra de adolescentes. Acción psicológica, (12), 1-14.

Serrano, D., Garrido, M., Notario, B., Bartolomé, R., Solera, M. y Martínez, V. (2012). Validez de la escala de Resiliencia de Connor-Davidson (CD-RISC) en una población de mayores entre 60 y 75 años. International Journal of psychological research, (5), 49-57. 